Research Article

\title{
Matrix Expression of Shapley Value in Graphical Cooperative Games
}

\author{
Yuanhua Wang $\mathbb{D}^{1},{ }^{1}$ Fuad E. Alsaadi $\mathbb{D},{ }^{2}$ Zheng Liu, ${ }^{1}$ Xiaomeng Wu, ${ }^{1}$ and Xiyu Liu $\mathbb{D}^{1}$ \\ ${ }^{1}$ Business School, Shandong Normal University, Ji'nan 250014, China \\ ${ }^{2}$ Department of Electrical and Computer Engineering, Faculty of Engineering, King Abdulaziz University, \\ Jeddah 21589, Saudi Arabia \\ Correspondence should be addressed to Yuanhua Wang; wyh_1005@163.com
}

Received 11 March 2020; Accepted 31 March 2020; Published 22 May 2020

Guest Editor: Rongwei Guo

Copyright (c) 2020 Yuanhua Wang et al. This is an open access article distributed under the Creative Commons Attribution License, which permits unrestricted use, distribution, and reproduction in any medium, provided the original work is properly cited.

\begin{abstract}
This paper studies a class of cooperative games, called graphical cooperative games, where the internal topology of the coalition depends on a prescribed communication graph among players. First, using the semitensor product of matrices, the value function of graphical cooperative games can be expressed as a pseudo-Boolean function. Then, a simple matrix formula is provided to calculate the Shapley value of graphical cooperative games. Finally, some practical examples are presented to illustrate the application of graphical cooperative games in communication-based coalitions and establish the significance of the Shapley value in different communication networks.
\end{abstract}

\section{Introduction}

Game theory provides a formal mathematical formation to describe the complex interactions among rational players [1]. In general, game theory can be divided into two branches: noncooperative games and cooperative games. In noncooperative game theory, the fundamental unit of study is the individual player, and the theory studies the strategic choices in the interactions among competing players. By contrast, the fundamental unit in cooperative game theory is the set of players or coalition, and the theory studies the behaviour of rational players when they cooperate. The fundamental problem in cooperative game theory is how to allocate the profit or value of a coalition to its individual players in such a way that players are encouraged to cooperate. A fair allocation that has been widely used is the Shapley value [2].

In recent years, there has been an increasing amount of research on using graph theory to cooperative games, where the players are interconnected and communicate with others in a network graph. For modelling the communication graph, cooperative games in the graph form, called graphical cooperative games or graphical coalitional games, are introduced by Myerson in [3]. These are games where the internal structure of the coalition is described by a network. In the network, nodes represent players and two players are interpreted to have an edge between them if and only if they can communicate with each other. In such games, unlike the value of a coalition depending solely on the members of that coalition in canonical cooperative games, the value function will be dependent of how the players are interconnected within the coalition. In [4-6], the formation and stability of coalitions given an underlying communication graph are studied, and its applications to communication networks have been investigated. In [7], the graphical coalitional games are studied with applications to economic and social networks, and the authors have analyzed the stability of networks when players can choose to form and maintain the links between them. This game model is also used to study biologic networks [8] and so on.

It is pointed out in [3] that the Shapley value is the only possible function that provides a fair allocation in graphical coalitional games. However, its computational complexity becomes an obstacle both in practical applications and 
theoretical deductions [9]; a remark is also presented in [6] to state that "the Shaple value is computationally expensive, but for fairly large structures one time computation is still affordable. For such a graph that even one time computation is not affordable, approximations can be used for its computation." In our recent work [10], a simple matrix formula is obtained to calculate the Shapley value and the basic mathematical method is the semitensor product of matrices (STP), which is a generalization of the traditional matrix product [11]. This method has been successfully applied in the analysis and control problems of logical networks [12-25], and then it has been used in finite game theory [26-30], evolutionary games [31-38], finite automata $[39,40]$, graph theory [41], etc. Unlike the canonical cooperative games being considered in [10], this paper studies a new class of cooperative games, called graphical cooperative games. Wang et al. [10] have provided the matrix formulas to calculate the symmetric and weighted Shapley values for canonical cooperative $n$-person games. However, this paper proposes a simple formula to calculate the Shapley value of graphical cooperative games, and it is more computationally complex than the formulas in [10].

The paper is organized as follows. A brief review for the STP method and graphical cooperative games is presented in Section 2. In Section 3, the matrix formula of the Shapley value in graphical cooperative games is presented. Section 4 discusses the practical applications of graphical cooperative games to some communication networks. Section 5 is a brief conclusion.

Before ending this section, we present some notations used in this paper. $\mathscr{D}:=\{0,1\} . \Delta_{n}:=\left\{\delta_{n}^{i} \mid i=1, \cdots, n\right\}$, where $\delta_{n}^{i}$ is the $i$ th column of the identity matrix $I_{n}$. $\mathbb{R}^{n}$ is the $n$ dimensional Euclidean space. $\mathscr{M}_{m \times n}$ denotes the set of $m \times$ $n$ real matrices. $\operatorname{Col}(M)(\operatorname{Row}(M))$ is the set of columns (rows) of $M$ and $\operatorname{Col}_{\mathrm{i}}(M)\left(\operatorname{Row}_{i}(M)\right)$ is the $i$ th column (row) of $M . \mathbf{1}_{\ell}=\underbrace{(1,1, \ldots, 1)}_{\ell}{ }^{T}$. A matrix $L \in \mathscr{M}_{m \times n}$ is called a logical matrix if the columns of $L$ are of the form of $\delta_{m}^{k}$, that is, $\operatorname{Col}(L) \subset \Delta_{m}$. Denote by $\mathscr{L}_{m \times n}$ the set of $m \times n$ logical matrixes.

\section{Preliminaries}

2.1. Semitensor Product of Matrices. The basic technique used in this paper is the STP of matrices. We can refer to [11] for details.

Definition 1. Let $M \in \mathscr{M}_{m \times n}, N \in \mathscr{M}_{p \times q}$, and $t=\operatorname{lcm}\{n, p\}$ be the least common multiple of $n$ and $p$. The STP of $M$ and $N$ is defined as

$$
M \propto N:=\left(M \otimes I_{t / n}\right)\left(N \otimes I_{t / p}\right) \in \mathscr{M}_{m t / n \times q t / p},
$$

where $\otimes$ is the Kronecker product. As a generalization of the conventional matrix product, the STP of matrices keeps the useful properties of the conventional matrix product available. For convenience, the symbol " $\propto$ " may be omitted without confusion.

Next, we present some properties of the STP used in this paper.
Proposition 1. Let $X \in \mathbb{R}^{m}$ be a column and $M$ be a matrix. Then,

$$
X \propto M=\left(I_{m} \otimes M\right) X
$$

The STP has pseudocommutative properties, and the swap matrix is defined as follows.

Definition 2 The matrix $W_{[m, n]} \in \mathscr{M}_{m n \times m n}$, defined by

$$
\begin{aligned}
W_{[m, n]}= & \delta_{m n}[1, m+1, \cdots,(n-1) m+1 ; 2, m \\
& +2, \ldots,(n-1) m+2 ; \ldots ; m, 2 m, \ldots, n m]
\end{aligned}
$$

is called the $(m, n)$ th dimensional swap matrix.

Proposition 2. Let $X \in \mathbb{R}^{m}$ and $Y \in \mathbb{R}^{n}$ be two columns. Then,

$$
W_{[m, n]} \propto X \propto Y=Y \propto X .
$$

If $x$ is a logical variable, then $x \in \mathscr{D}$. To use vector expression of logical values, we identify $1 \sim \delta_{2}^{1} \in \Delta$ and $0 \sim \delta_{2}^{2} \in \Delta$. Now, a pseudological (or logical) function can be expressed as an algebraic form.

Theorem 1. Let $f: \mathscr{D}^{n} \longrightarrow \mathbb{R}$ (or $\left.f: \mathscr{D}^{n} \longrightarrow \mathscr{D}\right)$ be a pseudological (or logical) function. Then, there exists a unique vector (or matrix) $M_{f} \in \mathbb{R}^{1 \times 2^{n}}$ (or $M_{f} \in \mathscr{L}_{2 \times 2^{n}}$ ), such that

$$
f\left(x_{1}, x_{2}, \ldots, x_{n}\right)=M_{f} \propto_{i=1}^{n} x_{i},
$$

where $M_{f}$ is called the structure vector (or matrix) of $f$, which can be calculated as

$$
\operatorname{Col}_{j}\left(M_{f}\right)=f\left(\delta_{k^{n}}^{j}\right), \quad j=1,2, \cdots, k^{n} .
$$

2.2. Graphical Cooperative Games. In essence, a canonical cooperative game involves a set of players, denoted by $N=\{1,2, \ldots, n\}$, who seek to form cooperative groups in order to strengthen their positions in the game. Any subset of $N$ is called a coalition, and $N$ is called the grand coalition. The second fundamental concept of the canonical cooperative game is the characteristic function or value function, denoted by $v: 2^{N} \longrightarrow \mathbb{R}$ with $v(\varnothing)=0$, which quantifies the worth of a coalition in a game. Therefore, a canonical cooperative game can be denoted by a pair $(N, v)$ [4]. In general, we can identify a cooperative game $(N, v)$ with its value function $v$, since for every $v$ a different game may be defined.

Next, we give the definition of graphical cooperative games.

Definition 3 (see [6]). A graphical cooperative game is denoted by $\Gamma=(G, v)$, where

(1) $G=(V, E)$ is an undirected graph with $V=\{1,2, \ldots, n\}$ a finite nonempty set of players and $E \subseteq V \times V$ a set of edges. The elements of $V$ are also called nodes or vertices. The number of elements in $V$ is called the order or size of $G$ and is denoted as 
$|G|=n$. In this paper, we only consider that $G$ is a simple graph, that is, $G$ does not contain self-loops and multiple edges. Let $S=\left(V^{\prime}, E^{\prime}\right)$ be a graph such that $V^{\prime} \subseteq V$ and $E^{\prime} \subseteq E$; then, $S$ is called a subgraph of $G$. If $E^{\prime}$ contains all the edges $e=\{i, j\} \in E$ with $i, j \in V^{\prime}$, then $S$ is called an induced subgraph of $G$ and denoted as $S \subseteq G$.

(2) $v: 2^{G} \longrightarrow \mathbb{R}$ is called the value function or characteristic function with $v(\varnothing)=0$, where $2^{G}$ is the collection of all the induced subgraphs of $G$. It should be noted that $v$ is required to satisfy four axioms of value [6].

For every induced subgraph $S \subseteq G$, that is, $S \in 2^{G}$, the value function $v(S)$ can be expressed as a pseudological function $v: \mathscr{D}^{n} \longrightarrow \mathbb{R}$ by using the logical variables; then, we have

$$
v(S)=v\left(x_{1}^{S}, x_{2}^{S}, \cdots, x_{n}^{S}\right), \quad S \in 2^{G},
$$

where $x_{i}^{S} \in \mathscr{D}$ is a logical variable and

$$
x_{i}^{S}= \begin{cases}1, & i \in S \\ 0, & i \notin S .\end{cases}
$$

Define a lexicographic order as

$$
T \prec S \longleftrightarrow d(T)>d(S),
$$

where $d(R)=\sum_{i=1}^{n} 2^{n-i} x_{i}^{R}$ is a decimal number corresponding to each induced subgraph $R \in 2^{G}$, and its binary number is $b(R)=x_{1}^{R} x_{2}^{R} \cdots x_{n}^{R}$. That is, we can describe $b\left(R_{1}\right)=11 \cdots 11, \quad b\left(R_{2}\right)=11 \cdots 10, \quad b\left(R_{3}\right)=11 \cdots 01$, $\cdots, b\left(R_{2^{n}}\right)=00 \cdots 00$.

Using Theorem 1, for every pseudological function $v$, we can find its structure vector $C_{v} \in \mathbb{R}^{2^{n}}$, such that equation (7) can be expressed into its matrix form as

$$
v(S)=C_{v} x^{S}
$$

where $\quad x^{S}=\propto_{i=1}^{n} x_{i}^{S} \in \Delta_{2^{n}} \quad$ and $\quad C_{v}:=\left[v\left(b\left(R_{1}\right)\right)\right.$, $\left.v\left(b\left(R_{2}\right)\right), \ldots, v\left(b\left(R_{2^{n}}\right)\right)\right]$. Because of $v(\varnothing)=0$, the last element of $C_{v}$ is 0 , that is, $v\left(b\left(R_{2^{n}}\right)\right)=0$.

\section{Matrix Formula of the Shapley Value in Graphical Cooperative Games}

In this section, we give a matrix formula to calculate the Shapley value in graphical cooperative games in order to reduce the computational complexity. First, we define the Shapley value of player $i$ in graphical cooperative games.

Definition 4 (see [6]). Consider a graphical cooperative game $\Gamma=(G, v)$; the Shapley value of player $i$ is defined as

$$
\varphi_{v}^{G}(i)=\frac{1}{|G|} \sum_{S \subseteq G \backslash\{i\}} \frac{(v(S \cup\{i\})-v(S))}{\left(\begin{array}{c}
|G|-1 \\
|S|
\end{array}\right)}, \quad i=1,2, \cdots, n,
$$

where $S \subseteq G \backslash\{i\}$ means $S$ is an induced subgraph of $G \backslash\{i\}$, and $S \cup\{i\}$ is an induced subgraph of $G$ that contains all the players in $S$ and player $i$.

Since $|G|=n$, then we have

$$
\left(\begin{array}{c}
|G|-1 \\
|S|
\end{array}\right)=C_{n-1}^{|S|}=\frac{(n-1) !}{|S| !(n-1-|S|) !}
$$

Plugging it into (11) yields

$$
\begin{aligned}
\varphi_{v}^{G}(i) & =\sum_{S \subseteq G \backslash\{i\}} \frac{|S| !(n-1-|S|) !}{n(n-1) !}[v(S \cup\{i\})-v(S)] \\
& =\sum_{S \subseteq G \backslash\{i\}} \frac{|S| !(n-1-|S|) !}{n !}[v(S \cup\{i\})-v(S)] .
\end{aligned}
$$

According to (10), we have the matrix expression as

$$
\begin{aligned}
v(S \cup\{i\})-v(S) & =C_{v}\left(x_{1}^{S} \cdots x_{i-1}^{S}\left[\begin{array}{l}
1 \\
0
\end{array}\right] x_{i+1}^{S} \cdots x_{n}^{S}-x_{1}^{S} \cdots x_{i-1}^{S}\left[\begin{array}{l}
0 \\
1
\end{array}\right] x_{i+1}^{S} \cdots x_{n}^{S}\right) \\
& =C_{v}\left(W_{\left[2,2^{i-1}\right]}\left[\begin{array}{l}
1 \\
0
\end{array}\right] x_{1}^{S} \cdots x_{i-1}^{S} x_{i+1}^{S} \cdots x_{n}^{S}-W_{\left[2,2^{i-1}\right]}\left[\begin{array}{l}
0 \\
1
\end{array}\right] x_{1}^{S} \cdots x_{i-1}^{S} x_{i+1}^{S} \cdots x_{n}^{S}\right) \\
& =C_{v}\left(W_{\left[2,2^{i-1}\right]}\left[\begin{array}{c}
1 \\
-1
\end{array}\right] x_{1}^{S} \cdots x_{i-1}^{S} x_{i+1}^{S} \cdots x_{n}^{S}\right),
\end{aligned}
$$


where $\ltimes_{j \neq i}^{n j=1} x_{j}^{S} \in \Delta_{2^{n-1}}$, and in vector form,

$$
x_{j}^{S}= \begin{cases}\delta_{2}^{1}, & j \in S, \\ \delta_{2}^{2}, & j \notin S .\end{cases}
$$

It follows that

$$
x_{1}^{S} \cdots x_{i-1}^{S} x_{i+1}^{S} \cdots x_{n}^{S}=\delta_{2^{n-1}}^{j}, \quad j=1,2, \cdots, 2^{n-1} .
$$

According to Definition 2, we have

$$
W_{\left[2,2^{i-1}\right]}\left[\begin{array}{c}
1 \\
-1
\end{array}\right]=\operatorname{diag}(\underbrace{\left[\begin{array}{c}
1 \\
-1
\end{array}\right], \cdots,\left[\begin{array}{c}
1 \\
-1
\end{array}\right]}_{2^{i-1}}) \in \mathscr{M}_{2^{i} \times 2^{i-1}} .
$$

Then, we obtain that

$$
W_{\left[2,2^{i-1}\right]}\left[\begin{array}{c}
1 \\
-1
\end{array}\right] \delta_{2^{n-1}}^{j}=\left(W_{\left[2,2^{i-1}\right]}\left[\begin{array}{c}
1 \\
-1
\end{array}\right] \otimes I_{2^{n-i}}\right) \delta_{2^{n-1}}^{j}=\operatorname{Col}_{j}\left(T_{i}\right)
$$

where

$$
\begin{aligned}
T_{i} & =\left(W_{\left[2,2^{i-1}\right]}\left[\begin{array}{c}
1 \\
-1
\end{array}\right]\right) \otimes I_{2^{n-i}} \\
& =\operatorname{diag}(\underbrace{\left[\begin{array}{c}
I_{2^{n-i}} \\
-I_{2^{n-i}}
\end{array}\right], \cdots,\left[\begin{array}{c}
I_{2^{n-i}} \\
-I_{2^{n-i}}
\end{array}\right]}_{2^{i-1}}) \in \mathscr{M}_{2^{n} \times 2^{n-1}} .
\end{aligned}
$$

Similar to the construction in [10], we construct a vector $\ell_{j}$ as follows:

$$
\left\{\begin{array}{l}
\ell_{1}=\left[\begin{array}{l}
1 \\
0
\end{array}\right], \\
\ell_{k+1}=\left[\begin{array}{c}
\ell_{k}+\mathbf{1}_{2^{k}} \\
\ell_{k}
\end{array}\right] \in \mathbb{R}^{2^{k+1}}, \quad k=1,2,3, \cdots .
\end{array}\right.
$$

Let $|S|=\left|\delta_{2^{n-1}}^{\alpha}\right|=\ell_{n-1}^{\alpha}, \quad \alpha=1,2, \cdots, 2^{n-1}$, where $\ell_{n-1}^{\alpha}$ denotes the $\alpha$ th element of $\ell_{n-1}$. Use $\ell_{n-1}$ to construct a new column vector $\eta_{n-1}=\left[\eta_{n-1}^{1}, \eta_{n-1}^{2}, \cdots, \eta_{n-1}^{2^{n-1}}\right]^{T}$, where

$$
\eta_{n-1}^{\alpha}=\left(\ell_{n-1}^{\alpha}\right) !\left(n-1-\ell_{n-1}^{\alpha}\right) !, \quad \alpha=1,2, \cdots, 2^{n-1} .
$$

Set $\xi=\eta_{n-1}$, and we divide $\xi$ into $k$ equal blocks as follows:

$$
\xi=\left[\xi_{k}^{1}, \xi_{k}^{2}, \cdots, \xi_{k}^{k}\right]^{T}, \quad k=1,2,2^{2}, \cdots, 2^{n-1}
$$

That is, when $k=2$, then $\xi$ can be divided into two equal blocks $\xi=\left[\begin{array}{l}\xi_{2}^{1} \\ \xi_{2}^{2}\end{array}\right], \ldots$, and when $k=2^{n-1}$, then $\xi=\left[\xi_{2^{n-1}}^{1}, \xi_{2^{n-1}}^{2}, \cdots, \xi_{2^{n-1}}^{2^{n-1}}\right]^{T}$.

Based on the abovementioned derivation, the Shapley value (13) can be written as

$$
\begin{aligned}
\varphi_{v}^{G}(i) & =\frac{1}{n !} \sum_{j=1}^{2^{n-1}} C_{v} \eta_{n-1}^{j} W_{\left[2,2^{i-1}\right]}\left[\begin{array}{c}
1 \\
-1
\end{array}\right] \delta_{2^{n-1}}^{j} \\
& =\frac{1}{n !} C_{v} \cdot \sum_{j=1}^{2^{n-1}} \eta_{n-1}^{j} \operatorname{Col}_{j}\left(T_{i}\right) \\
& =\frac{1}{n !} C_{v} \cdot\left[\begin{array}{c}
\xi_{2^{i-1}}^{1} \\
-\xi_{2^{i-1}}^{1} \\
\xi_{2^{i-1}}^{2} \\
-\xi_{2^{i-1}}^{2} \\
\vdots, \\
\xi_{2^{i-1}}^{2^{-1}} \\
-\xi_{2^{i-1}}^{2^{i-1}}
\end{array}\right], \quad i=1,2, \cdots, n .
\end{aligned}
$$

Then, we have the following result for calculating the Shapley value in graphical cooperative games.
Theorem 2. Consider a graphical cooperative game $\Gamma=(G, v)$, its Shapley value can be calculated by the following matrix formula: 


$$
\varphi_{v}^{G}=\left[\varphi_{v}^{G}(1) \varphi_{v}^{G}(2) \cdots \varphi_{v}^{G}(n)\right]=\frac{1}{n !} C_{v} \cdot \Xi
$$

where

$$
\Xi=\left[\begin{array}{cccc} 
& & & \xi_{2^{n-1}}^{1} \\
& & & -\xi_{2^{n-1}}^{1} \\
& \xi_{2}^{1} & & \xi_{2^{n-1}}^{2} \\
\xi_{1}^{1} & -\xi_{2}^{1} & \cdots & -\xi_{2^{n-1}}^{2} \\
-\xi_{1}^{1} & \xi_{2}^{2} & & \vdots \\
& -\xi_{2}^{2} & & \xi_{2^{n-1}}^{2^{n-1}} \\
& & & -\xi_{2^{n-1}}^{2^{n-1}}
\end{array}\right] \in M_{2^{n} \times n}
$$

Remark 1. In the conversional formula of the Shapley value (13), the computational complexity is $\mathcal{O}\left(n !^{2}\right)$. Using the STP method, a simple matrix form of the Shapley value is provided in Theorem 2. Observing (24), in order to calculate the Shapley value, we just need to discuss the computational complexity of matrix $\Xi$. Obviously, the dimension of $\Xi$ is $2^{n} \times n$ and with the help of MATLAB, the matrix $\Xi$ can be easily obtained by using the recursive algorithm, which is the major advantage of Theorem 2 .

\section{Practical Examples of Graphical Cooperative Games}

In this section, we consider some practical examples of graphical cooperative games, which are presented in [6]. These examples show the applications of graphical cooperative games to communication network of sensor nodes. Nodes communicate with each other through links.

Example 1. Assume that the value of a communication network is the number of nodes pairs which can communicate with each other; then, the value function for a connected graph of size $n$ is

$$
v_{n}= \begin{cases}C_{n}^{2}, & n>1, \\ 0, & n=1 .\end{cases}
$$

Three different communication networks between six nodes are shown in Figure 1.

Using (10), the value function of graph $G_{a}$ shown in Figure 1(a) can be expressed as

$$
v_{G_{a}}(S)=C_{v}^{G_{a}} \propto \propto_{i=1}^{6} x_{i}^{S},
$$

where $C_{v}^{G_{a}} \in \mathbb{R}^{2^{6}}$ is a row vector; using (26), it is easy to calculate that
$C_{v}^{G_{a}}=[64434333422121113110100031101000$

$$
42212111311010003110100031101000] \text {. }
$$

Then, we construct a column vector $\ell_{5} \in \mathbb{R}^{2^{5}}$ as $\ell_{5}=[54434332433232214332322132212110]^{T}$.

According to (21), a new column vector $\xi$ can be calculated as

$$
\begin{gathered}
\xi=\left[\begin{array}{ll}
1202424 & 12241212122412121212121224 \\
241212121212122412121224122424 & 120
\end{array}\right]^{T} .
\end{gathered}
$$

Now, we divide $\xi$ into $k$ equal blocks, where $k=1,2,2^{2}, 2^{3}, 2^{4}, 2^{5}$.

Using Theorem 2, we have

$$
\Xi=\left[\begin{array}{cccccc}
120 & 120 & 120 & 120 & 120 & 120 \\
24 & 24 & 24 & 24 & 24 & -120 \\
24 & 24 & 24 & 24 & -120 & 24 \\
12 & 12 & 12 & 12 & -24 & -24 \\
24 & 24 & 24 & -120 & 24 & 24 \\
12 & 12 & 12 & -24 & 12 & -24 \\
12 & 12 & 12 & -24 & -24 & 12 \\
12 & 12 & 12 & -12 & -12 & -12 \\
\vdots & \vdots & \vdots & \vdots & \vdots & \vdots \\
-12 & -12 & -12 & 12 & 12 & 12 \\
-12 & -12 & -12 & 24 & 24 & -12 \\
-12 & -12 & -12 & 24 & -12 & 24 \\
-24 & -24 & -24 & 120 & -24 & -24 \\
-12 & -12 & -12 & -12 & 24 & 24 \\
-24 & -24 & -24 & -24 & 120 & -24 \\
-24 & -24 & -24 & -24 & -24 & 120 \\
-120 & -120 & -120 & -120 & -120 & -120
\end{array}\right] \in M_{2^{6} \times 6} .
$$

According to (24), we can easily obtain its Shapley value by using MATLAB software, which is presented as

$$
\varphi_{v}^{G_{a}}=\frac{1}{6 !} C_{v}^{G_{a}} \cdot \Xi=\left[\begin{array}{llllll}
0.833 & 1.333 & 0.833 & 1 & 1 & 1
\end{array}\right] .
$$

Similarly, the Shapley value of each node in Figures 1(b) and $1(\mathrm{c})$ can also be given by 


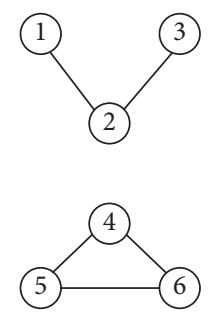

(a)

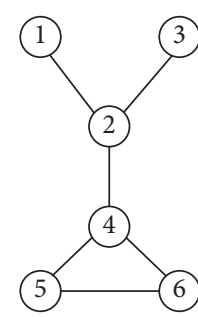

(b)

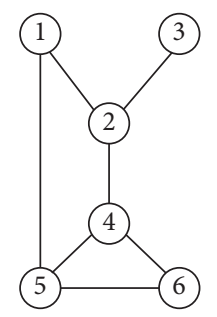

(c)

FIgURE 1: Communication network graphs.

$\varphi_{v}^{G_{b}}=\frac{1}{6 !} C_{v}^{G_{b}} \cdot \Xi=\left[\begin{array}{llllll}1.667 & 4.167 & 1.667 & 3.833 & 1.833 & 1.833\end{array}\right]$,

$\varphi_{v}^{G_{c}}=\frac{1}{6 !} C_{v}^{G_{c}} \cdot \Xi=\left[\begin{array}{llllll}2.35 & 3.683 & 1.75 & 2.65 & 2.517 & 2.05\end{array}\right]$, where
It is shown that, in three network graphs, the only difference is the structure vector of value function, which is dependent of its corresponding communication graph. Therefore, for a communication network with the same number of nodes, the matrix $\Xi$ is the same. Besides, it is to be noted that our computational results coincide with the values presented in [6].

Based on the above calculated Shapley values, it can be seen that the more a node is involved in the communication network, the more is its Shapley value. For example, in Figure 1(a), node 2 is more vital than any of the nodes 4,5 , and 6 , since node 2 exclusively supports the communication between nodes 1 and 3. Comparing Figures 1(b) and 1(c), the addition of new link between nodes 1 and 5 in Figure 1(c) can support the communication between nodes 1 and 5, which is exclusively supported by the link between nodes 2 and 4 in Figure 1(b); hence, it assigns more Shapley values to nodes 2 and 4 in Figure 1(b).
The following example presented in [6] shows that, for some communication networks with special structure topologies, the Shapley value can be simple to calculate regardless of the number of players.

Example 2. Consider a network with $n$ nodes, the structure topology between them is a star graph with node 1 as the star point. In [6], a simple formula to compute the Shapley value of each node is given and this formula holds for any number $n$ of nodes. Using our matrix formula (24) in Theorem 2, it is also easy to calculate the Shapley value, and it is shown that, for the star graph between $n$ nodes, the computational complexity between the formula in [6] and our matrix formula (24) is the same. Moreover, it is obvious that, in a star graph for the structure vector $C_{v}$ of value function in (10), the last $\mathbb{R}^{2^{n-1}}$ elements of $C_{v}$ are all 0 , since $x_{1}^{S}=0$. For example, when $n=6$, the network graph is described in Figure 2, and its structure vector can be calculated that

$$
C_{v}=[15,10,10,6,10,6,6,3,10,6,6,3,6,3,3,1,10,6,6,3,6,3,3,1,6,3,3,1,3,1,1,0,0, \cdots, 0] \in \mathbb{R}^{2^{6}}
$$

Using (24), where $\Xi$ is the same as in example 1 , we have its Shapley value as

$$
\left\{\begin{array}{l}
\varphi_{v}^{G}(1)=5.833 \\
\varphi_{v}^{G}(i)=1.833, \quad i=2, \cdots, 6
\end{array}\right.
$$




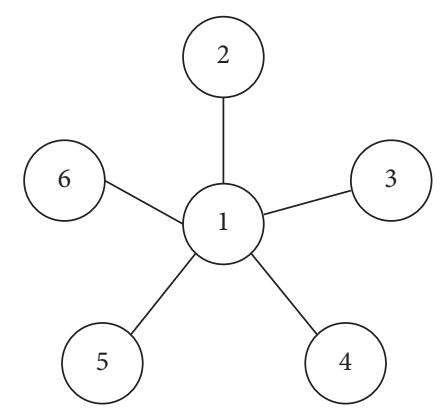

FIgURE 2: Star graph with six nodes.

It is established that node 1 is the most vital node in this communication graph.

From these two simple examples, it is worth noting the advantage of our result in Theorem 2. Regardless of the structure topology for communication networks, we only need to calculate the matrix $\Xi$, which is easily obtained by using recursive algorithm with the help of MATLAB. However, it is pointed in [6] that the Shapey value is computationally expensive and for a fairly large graph even one time computation is not affordable.

\section{Conclusion}

This paper considers a new class of cooperative games, called graphical cooperate games. Using the STP method, the value function is presented as a pseudo-Boolean function, and a corresponding formula is obtained to calculate the Shapley value for graphical cooperate games. Then, the worth of each player in a coalition based on its connectivity in communication network can be explored. Some examples are given to demonstrate the theoretical results. In our future work, we will study more useful properties of graphical cooperative games based on the Shapley value.

With the rapid development of science, the game-theoretic approach will be a promising new method for studying more control problems in the future, such as synchronization [42-49], and consensus problems [50-53].

\section{Data Availability}

No data were used to support this study.

\section{Conflicts of Interest}

The authors declare that they have no conflicts of interest.

\section{Acknowledgments}

This work was supported by the National Natural Science Foundation (NNSF) of China under Grants 61903236, 61876101, 61773371, and 71701115.

\section{References}

[1] J. Von Neumann and O. Morgenstern, Theory of Games and Economic Behavior, Princeton University Press, Princeton, NJ, USA, 1944.
[2] L. S. Shapley, "17. A value for n-person games," Contributions to the Theory of Games (AM-28), Volume II, vol. 2, no. 28, pp. 307-318, 1953.

[3] R. B. Myerson, "Graphs and cooperation in games," Mathematics of Operations Research, vol. 2, no. 3, pp. 225-229, 1977.

[4] W. Saad, Z. Han, M. Debbah et al., "Coalitional game theory for communication networks: a tutorial," IEEE Signal Processing Magazine, vol. 26, no. 5, pp. 77-97, 2009.

[5] W. Saad, Z. Han, T. Başar et al., "Coalition formation games for collaborative spectrum sensing," IEEE Transactions on Vehicular Technology, vol. 60, no. 1, pp. 276-297, 2011.

[6] M. Aurangzeb and F. L. Lewis, "Internal structure of coalitions in competitive and altruistic graphical coalitional games," Automatica, vol. 50, no. 2, pp. 335-348, 2014.

[7] M. O. Jackson and A. Watts, "The evolution of social and economic networks," Journal of Economic Theory, vol. 106, no. 2, pp. 265-295, 2002.

[8] S. Moretti, V. Fragnelli, F. Patrone et al., "Using coalitional games on biological networks to measure centrality and power of genes," Bioinformatics, vol. 26, no. 21, pp. 2721-2730, 2010.

[9] J. R. Marden and A. Wierman, "Distributed welfare games," Operations Research, vol. 61, no. 1, pp. 155-168, 2013.

[10] Y. Wang, D. Cheng, and X. Liu, "Matrix expression of Shapley values and its application to distributed resource allocation," Science China Information Sciences, vol. 62, no. 2, pp. 22201 : 1-11, 2019.

[11] D. Cheng, H. Qi, and Z. Li, Analysis and Control of Boolean Networks - A Semi-tensor Product Approach, Springer, London, UK, 2011.

[12] H. Li, X. Xu, and X. Ding, "Finite-time stability analysis of stochastic switched Boolean networks with impulsive effect," Applied Mathematics and Computation, vol. 347, pp. 557-565, 2019.

[13] J. Zhong, Y. Liu, K. I. Kou, L. Sun, and J. Cao, "On the ensemble controllability of Boolean control networks using STP method," Applied Mathematics and Computation, vol. 358, pp. 51-62, 2019.

[14] Y. Liu, Y. Zheng, H. Li, F. E. Alsaadi, and B. Ahmad, "Control design for output tracking of delayed Boolean control networks," Journal of Computational and Applied Mathematics, vol. 327, pp. 188-195, 2018.

[15] Q. Zhu, Y. Liu, J. Lu et al., "Further results on the controllability of Boolean control networks," IEEE Transactions on Automatic Control, vol. 64, no. 1, pp. 440-442, 2019.

[16] J. Lu, J. Zhong, C. Huang et al., "On pinning controllability of Boolean control networks," IEEE Transactions on Automatic Control, vol. 61, no. 6, pp. 1658-1663, 2016.

[17] H. Li, L. Xie, and Y. Wang, "Output regulation of Boolean control networks," IEEE Transactions on Automatic Control, vol. 62, no. 6, pp. 2993-2998, 2017.

[18] J. Lu, H. Li, Y. Liu et al., "Survey on semi-tensor product method with its applications in logical networks and other finite-valued systems," IET Control Theory \& Applications, vol. 11, no. 13, pp. 2040-2047, 2017.

[19] Y. Li, H. Li, and W. Sun, "Event-triggered control for robust set stabilization of logical control networks," Automatica, vol. 95, pp. 556-560, 2018.

[20] X. Xu, Y. Liu, H. Li et al., "Robust set stabilization of Boolean control networks with impulsive effects," Nonlinear Analysis: Modelling and Control, vol. 23, no. 4, pp. 553-567, 2018.

[21] G. Zhao and S. Fu, "Matrix approach to trajectory control of higher-order k-valued logical control networks," IET Control Theory \& Applications, vol. 11, no. 13, pp. 2110-2115, 2017. 
[22] Y. Li, H. Li, and X. Ding, "Set stability of switched delayed logical networks with application to finite-field consensus," Automatica, vol. 113, 2020.

[23] Y. Zheng, H. Li, X. Ding, and Y. Liu, "Stabilization and set stabilization of delayed Boolean control networks based on trajectory stabilization," Journal of the Franklin Institute, vol. 354, no. 17, pp. 7812-7827, 2017.

[24] Y. Wang, X. Zhang, and Y. Hao, "Robust controllability and observability of boolean control networks under different disturbances," Mathematical Problems in Engineering, vol. 2019, Article ID 1813594, 2019.

[25] H. Li and Y. Wang, "Lyapunov-based stability and construction of Lyapunov functions for Boolean networks," SIAM Journal on Control and Optimization, vol. 55, no. 6, pp. 3437-3457, 2017.

[26] D. Cheng and T. Xu, "Application of STP to cooperative games," in Proceedings of the 10th IEEE ICCA, pp. 1680-1685, Zhejiang, China, 2013.

[27] D. Cheng, "On finite potential games," Automatica, vol. 50, no. 7, pp. 1793-1801, 2014.

[28] Y. Wang, T. Liu, and D. Cheng, "From weighted potential game to weighted harmonic game," IET Control Theory \& Applications, vol. 11, no. 13, pp. 2161-2169, 2017.

[29] H. Li, X. Ding, Q. Yang et al., "Algebraic formulation and Nash equilibrium of competitive diffusion games," Dynamic Games and Applications, vol. 8, no. 2, pp. 423-433, 2018.

[30] Y. Hao, S. Pan, Y. Qiao et al., "Cooperative control via congestion game approach," IEEE Transactions on Automatic Control, vol. 63, no. 12, pp. 4361-4366, 2018.

[31] H. Li, X. Ding, A. Alsaedi et al., "Stochastic set stabilization of n-person random evolutionary Boolean games and its applications," IET Control Theory \& Applications, vol. 11, no. 13, pp. 2152-2160, 2017.

[32] G. Zhao, H. Li, W. Sun, and F. E. Alsaadi, "Modelling and strategy consensus for a class of networked evolutionary games," International Journal of Systems Science, vol. 49, no. 12, pp. 2548-2557, 2018.

[33] P. Guo, H. Zhang, F. E. Alsaadi et al., "Semi-tensor product method to a class of event-triggered control for finite evolutionary networked games," IET Control Theory \& Applications, vol. 11, no. 13, pp. 2140-2145, 2017.

[34] X. Ding, H. Li, Q. Yang, Y. Zhou, A. Alsaedi, and F. E. Alsaadi, "Stochastic stability and stabilization of n -person random evolutionary Boolean games," Applied Mathematics and Computation, vol. 306, pp. 1-12, 2017.

[35] S. Fu, H. Li, G. Zhao et al., "Modelling and strategy optimisation for a kind of networked evolutionary games with memories under the bankruptcy mechanism," International Journal of Control, vol. 91, no. 5, pp. 1104-1117, 2018.

[36] G. Zhao, Y. Wang, and H. Li, "A matrix approach to the modeling and analysis of networked evolutionary games with finite memories," IEEE/CAA Journal of Automatica Sinica, vol. 5, no. 4, pp. 818-826, 2018.

[37] T. Liu, J. Wang, X. Zhang et al., "Game theoretic control of multiagent systems," SIAM Journal on Control and Optimization, vol. 57, no. 3, pp. 1691-1709, 2019.

[38] J. Wang, X. Gao, and Y. Xu, "Intermittent control for demand-side management of a class of networked smart grids," IET Control Theory \& Applications, vol. 13, no. 8, pp. 11661172, 2019.

[39] Y. Yan, Z. Chen, and Z. Liu, "Semi-tensor product approach to controllability and stabilizability of finite automata," Journal of Systems Engineering and Electronics, vol. 26, no. 1, pp. 134-141, 2015.
[40] W. Dou, H. Li, and F. E. Alsaadi, "Semitensor product approach to controllability, reachability, and stabilizability of probabilistic finite automata," Mathematical Problems in Engineering, vol. 2019, Article ID 8021750, 2019.

[41] J. Zhong, J. Lu, C. Huang, L. Li, and J. Cao, "Finding graph minimum stable set and core via semi-tensor product approach," Neurocomputing, vol. 174, pp. 588-596, 2016.

[42] E. Alzahrani, H. Akca, X. Li et al., "New synchronization schemes for delayed chaotic neural networks with impulses," Neural Computing and Applications, vol. 28, no. 9, pp. 2823-2837, 2017.

[43] R. Guo, "Projective synchronization of a class of chaotic systems by dynamic feedback control method," Nonlinear Dynamics, vol. 90, no. 1, pp. 53-64, 2017.

[44] X. Li, R. Rakkiyappan, and N. Sakthivel, "Non-fragile synchronization control for Markovian jumping complex dynamical networks with probabilistic time-varying coupling delays," Asian Journal of Control, vol. 17, no. 5, pp. 1678-1695, 2015.

[45] X. Xu, Y. Liu, H. Li et al., "Synchronization of switched Boolean networks with impulsive effects," International Journal of Biomathematics, vol. 11, no. 6, 2018.

[46] Z. Wang and R. Guo, "Hybrid synchronization problem of a class of chaotic systems by an universal control method," Symmetry, vol. 10, no. 11, p. 552, 2018.

[47] X. Li, P. duan, J. Cao et al., "Exponential synchronization of neural networks via feedback control in complex environment," Complexity, vol. 1, pp. 1-13, 2018.

[48] Y. Li, P. Duan, and H. Li, "Synchronization of switched logical control networks via event-triggered control," Journal of The Franklin Institute, vol. 355, no. 12, pp. 5203-5216, 2018.

[49] X. Zhang, X. Lv, and X. Li, "Sampled-data-based lag synchronization of chaotic delayed neural networks with impulsive control," Nonlinear Dynamics, vol. 90, no. 3, pp. 2199-2207, 2017.

[50] X. Tan, J. Cao, X. Li et al., "Leader-following mean square consensus of stochastic multi-agent systems with input delay via event-triggered control," IET Control Theory \& Applications, vol. 12, no. 2, pp. 299-309, 2018.

[51] Y. Li, H. Li, X. Ding, and G. Zhao, "Leader-follower consensus of multiagent systems with time delays over finite fields," IEEE Transactions on Cybernetics, vol. 49, no. 8, pp. 3203-3208, 2019.

[52] X. Tan, J. Cao, X. Li et al., "Consensus of leader-following multiagent systems: a distributed event-triggered impulsive control strategy," IEEE Transactions on Cybernetics, vol. 49, no. 3, pp. 792-801, 2019.

[53] Y. Li, H. Li, X. Xu et al., "A semi-tensor product approach to minimal-agent consensus control of networked evolutionary games," IET Control Theory \& Applications, vol. 12, no. 16, pp. 2269-2275, 2018. 